Prof. Dr. med. C. Bayerl im Gespräch mit Herrn Prof. Dr. Ralf Gutzmer:

\section{Die Bedeutung des Histamin H4-Rezeptors}

Prof. Dr. C. Bayerl: Lieber Herr Professor Gutzmer, wo im menschlichen Organismus und wo in der Haut finden wir den H4-Rezeptor (H4R)?

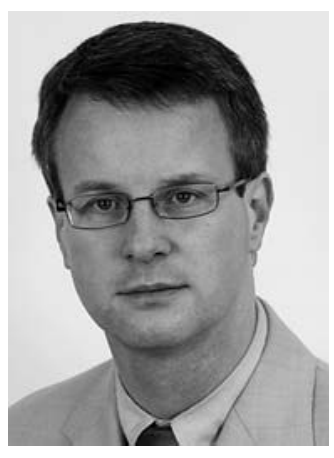

Prof. Dr. R. Gutzmer: Der H4R wird auf vielen menschlichen Zellen exprimiert, insbesondere auf Zellen des Immunsystems wie eosinophilen Granulozyten, Untergruppen von T-Zellen und Antigen-präsentierenden Zellen. Zur Expression in der Haut gibt es bislang nur wenige Daten, die eine Expression auf den Langerhans-Zellen und speziellen Antigen-präsentierenden Zellen gezeigt haben (den sogenannten IDEC, die in entzündeter Haut der atopischen Dermatitis anzufinden sind, und den plasmazytoiden dendritischen Zellen in der Psoriasis). Auch Keratinozyten scheinen den H4R auf ihrer Oberfläche zu tragen, die Funktion ist hier aber noch unklar.

Prof. Dr. C. Bayerl: Welche funktionelle Bedeutung hat der H4R? Prof. Dr. R. Gutzmer: Der H4R beeinflusst die Bewegung der Zellen (im Sinn einer Chemotaxis) sowie deren Fähigkeit, Botenstoffe des Immunsystems zu bilden. Auf humanen Zellen führt die Stimulation des H4R zu einer Herunterregulation von Chemokinen (wie CCL2) und Zytokinen (wie IL-12 und TNFa) und hat damit eine antiinflammatorische Wirkung, insbesondere bei Th1-dominierten Entzündungen. Auf T-Zellen führte die Stimulation des H4R zu einer Produktion des Th2-Zytokins IL-31, sodass der H4R eine Th2-dominierte Entzündung verstärken könnte.

Eine weitere wichtige Bedeutung des H4R ist die Induktion von Pruritus, was in verschiedenen Mausmodellen gezeigt werden konnte. Unklar ist bislang jedoch der Mechanismus, über den der H4R Juckreiz induziert.
Prof. Dr. C. Bayerl: Bei welchen Hauterkrankungen kann er als therapeutisches Ziel dienen?

Prof. Dr. R. Gutzmer: Die potenziellen Einsatzmöglichkeiten von H4R-Liganden im Bereich entzündlicher und juckender Hauterkrankungen sind vielfältig. So wird der Einsatz bei Ekzemerkrankungen, Asthma, Psoriasis und Autoimmunerkrankungen diskutiert. Juckende Hauterkrankungen mit Entzündung als auch ohne Entzündung (z.B. Prurigoerkrankungen, Pruritus bei internistischen Grunderkrankungen) stellen eine mögliche Indikation für eine H4R-gerichtete Therapie dar.

Prof. Dr. C. Bayerl: Welche H4R-Antagonisten sind entwickelt worden und welche werden für die Dermatologie in klinische Studien gehen? Für welche Indikation werden die ersten Studien starten?

Prof. Dr. R. Gutzmer: Verschiedene pharmazeutische Firmen haben H4R-Liganden in der präklinischen Entwicklung. Erste Phase-I-Studien am Menschen wurden durchgeführt, aber bislang nicht publiziert. Als erste Indikationen für frühe klinische Studien sind Asthma, atopische Dermatitis und Pruritus in der Diskussion. Bei der Entwicklung der Protokolle muss je nach Indikation sorgfältig abgewogen werden, ob eine topische oder systemische Applikation eines H4R-Agonisten oder -Antagonisten sinnvoll ist.

Prof. Dr. Ralf Gutzmer

Klinik für Dermatologie, Allergologie und Venerologie

Medizinische Hochschule Hannover

Ricklinger Str. 5

30449 Hannover

gutzmer.ralf@mh-hannover.de 